\title{
Was leistet die Röntgen-Durchleuchtung des Brustkorbes als Diagnostikum bei tuberkulösen Lungenerkrankungen?
}

Von

\author{
Dr. med. Georg Schellenberg, und Dr. med. August Seherer, \\ oberarzt an den Heilstätten Beelitz. \\ Chefarzt der Lungenheilstätte Mühltal bei \\ Bromberg, früher Oberarzt in Beelitz.
}

.Es bedurfte einer Reihe von Jahren, bis sich die Röntgenstrahlen unter den Untersuchungsmitteln der inneren Medizin eine gebührende Stellung erwarben. Ein energisches Zusammenarbeiten der Ärzteschaft und der elektrotechnischen Institute hat grosse Fortschritte. erzielt, die mit den Riederscben Momentaufnahmen des menschlichen Brustkorbes im Jahre 1899 allerseits volle Befriedigung gewähren mussten. Es hat vieler Arbeit bedurft, all die Zweifler und Gegner von der Bedeutung der Röntgenstrahlen für die innere Medizin $z u$ überzeugen und sie dahin zu bringen, dass sie der Röntgenuntersuchung einen vollberechtigten Platz unter den diagnostischen Hilfsmitteln einräumten.

Namentlich in Frankreich hat sich die medizinische Welt der Röntgenuntersuchung ganz besonders angenommen; ihre Resultate sind auch zum grössten Teil bestätigt worden. Grunmach und Rosenfeld zeigten im Jahre 1896 die Schattenbildungen älterer Lungenprozesse (Kalkeinlagerungen). Wassermann, Benedikt und $R u m p f$ bemühten sich um den röntgenologischen Nachweis von Kavernen. Schatten, jüngeren Verdichtungsherden entsprechend, beobachteten 1898 Béclère, Bauer und Immelmann; letzterer entdeckte mit dem Röntgenlicht einige Male Herde, die dann erst später klinisch nachgewiesen wurden. Rieders und Rosenthals Thorax-Momentanfnahmen in den Jahren 1899 nnd 1900 zeigten die 
Überlegenheit der Methode für Anfangsfälle mit zerstreuten einzelnen und tief liegenden Herden.

Eine Reihe von Universitätskliniken, Krankenhäusern und Privatinstituten ist heutzutage eifrig bemüht, die Röntgenmethode zu studieren und weiteren Kreisen bereitwilligst bekannt zu machen.

Vielfach ist auch die Durchleuchtungsmethode schon praktisch in Anwendung gekommen; es sind in Frankreich staatlicherseits Anstalten für Lungenuntersuchungen, namentlich der Wehrpflichtigen, mit der Absicht eingerichtet worden, Fälle, die über den Beginn hinaus, aber derzeit latent sind, auszuschalten (L é on Colin: Acad. de Méd. 24. X. 97).

Eine grosse Literatur ist bereits über die Verwendung der Röntgenstrahlen bei Lungentuberkulose, ganz besonders bei inzipienter Tuberkulose vorhanden; in ihr sind äusserst widersprechende Meinungen niedergelegt. Während die einen Autoren $(\mathrm{B} \mathrm{ouchard}, \mathrm{Brook}$ und Stanley Green, Gardiner) glauben, mit Sicherheit die Diagnose "Phthise" zu einer Zeit stellen zu können, zu der es mit den anderen klinischen Untersuchungsmitteln noch nicht gelingt, leisten die Röntgenstrahlen nach anderen (Hildebrand, $\mathrm{O}$ udin und Barthélémy) bei Spitzenaffektionen nicht mehr als die bisherigen Methoden. AlbersS chönberg äussert sich folgendermassen: „Es ist vielfach behauptet und ebenso oft bestritten, dass die Spitzenerkrankungen in einem Stadium nachweisbar sind, in dem Perkussion und Anskultation noch absolut keinen anatomischen Befund geben: der Beweis eines derartigen positiven röntgenologischen Befundes dürfte unter allen Umständen beim Fehlen sonstiger diagnostischer Merkmale recht schwer zu erbringen sein. Wenn man etwas geringe Ansprüche stellt, so wird man noch recht erfreuliche Resultate bei der Spitzenuntersuchung zu verzeichnen haben. Eine nur auf den röntgenologischen Befund aufgebaute Diagnose der Spitzenaffektionen bei fehlenden Befunden der Perkussion und Auskultation halte ich für unstatthaft." Einige Zeilen weiter sagt er: „Heutzutage sind wir noch nicht viel weiter, als dass wir die Röntgenmethode der Spitzenuntersuchung den anderen physikalischen Methoden als wertvolle Ergänzung in zweifelhaften Fällen anreihen können. “

Ähnlicher Ansicht ist auch Hildebrand. Er betont, dass die Lungenspitzengegenden für die Durchleuchtung sehr ungünstige Objekte sind. Er sagt: „Deutlich abgegrenzte Herde in den Lungenspitzen konnten, wenn sie sich mit Auskultation und Perkussion nicht hatten feststellen lassen, nie mit Sicherheit nachgewiesen werden; wurden Veränderungen gefunden, so waren auch klinische Symptome nachweisbar. Bei der geringen Dicke der Lunge an der Spitze lässt sich 
von vornherein nichts anderes erwarten. Die Herde müssen, um durch die Hilsmuskulatur hindurch einen Schatten zu geben, immerhin eine gewisse Ausdehnung haben, und wenn sie diese haben, so müssen sie auch für die Auskultation und Perkussion zugänglich sein, da sie sich nicht weit von der Oberfläche befinden. Das Röntgenbild bei Spitzenaffektionen leistet nicht mehr als die übrigen Untersuchungsmethoden. Bei zweifelhaften Fällen ist eine Röntgenuntersuchung nicht angebracht."

Mit der Diagnose der inzipienten Phthise befassten sich vor allem die Arbeiten von Barthélémy und Bouchard, Gardiner, Kelsch und Boinon, Leroux, Oudin, Walsham und Williams. Letzterer hat an 100 Tuberkulösen röntgenologische Untersuchungen angestellt und bei diesen Patienten, namentlich bei einseitigen oder überwiegend einseitigen Infiltrationen der Lunge, eine dunklere Fürbung der erkrankten Lungenpartie, einen inspiratorischen Hochstand des Zwerchfells beobachtet. Das Zurückbleiben des Zwerchfells auf der kranken Seite bei der Inspiration ist für jene Fälle wichtig, bei denen der lokale Prozess noch nicht zu Schatten gebenden Verdichtungsmassen herangewachsen ist. Willia ms erklärt das nach ihm benannte Symptom mit einer Kapazitätsveränderung der erkrankten Lange, die inspiratorisch eine geringere Volumenzunahme und daher eine schwächere Zwerchfellsbewegung zur Folge hat. Seine Methode versagte nur in zwei von 165 Fällen; dagegen hatten $\mathrm{Kelsch}$ und Boinon bei Durchleuchtung von 124 Fällen klinisch diagnostizierter Apicitis 73 in jeder Richtung negative Befunde. Gocht äussert sich ferner über den diagnostischen Wert der Röntgenstrahlen für die beginnende Tuberkulose folgendermassen: "Die Röntgenstrablen sind ein wesentliches Hilfsmittel für die Frühdiagnose der Phthisis pulmonum. So gut wie ausnahmslos lassen sich aut dem Schirm und auf der Platte bei klinisch diagnostizierter Spitzenerkrankung Schattendifferenzen feststellen. Die diffus erkrankte infiltrierte Spitze erscheint dunkler; zirkumskriptere Schatten, umgeben von normal durchlässigen Lungenpartien, lassen auf einzelne frischkranke Herde schliessen, falls alte verkalkte Herde klinisch ausgeschlossen werden können."

Wị haben bei der Durchleuchtung unserer Patienten ganz besonders auf die Lungenspitzenaffektionen geachtet. Infolge der mitunter stark auseinandergehenden Meinungen iiber den Wert der röntgenologischen Tuberkulosediagnostik gingen wir sehr kritisch zu Werke. Vor allen Dingen haben wir die Durchleuchtung unabhängig von der klinischen Untersuchung vorgenommen; der eine Kollege untersuchte die Fälle klinisch, der andere röntgenologisch. Es war absolut keine Beeinflussung von der einen Seite auf die andere mög- 
lich. Wir haben so im ganzen 320 Fälle durchleuchtet und werden im folgenden dieses reiche und vortreff liche Material einer eingehenden Betrachtung unterziehen. Die Kranken wurden zum grössten Teil kurz nach der Aufnahme, also der ersten klinischen Untersuchung, durchleuchtet; der Befund wurde aufgezeichnet und in vielen Fällen später noch einmal kontrolliert.

Wir müssen vorweg nehmen, dass wir eine vorzügliche Röntgeneinrichtung nach Walter mit dreiteiligem, elektrolytischen Unterbrecher nach Wehnelt von der Firma Reiniger, Gebbert und Schall zur Verfügung hatten und vor allen Dingen, was von ganz besonderer Wichtigkeit bei dieser Art von Untersuchungen ist, nicht allzu sparsam mit dem Röhrenmaterial umgehen mussten. Wir benützten Röhren von Re in i ger, Gebbert und Schall, Gundelach, Hirschmann; im grossen und ganzen waren wir immer mit dem gelieferten Material zufrieden. Wir bezogen die Röhren, mit Ausnahme der Hirschmannschen, von Reiniger, Gebbert und Schall.

Zunächst wollen wir im folgenden einige Punkte mehr technischer Art erörtern. Für eine gute Röntgendurchleuchtung ist ein absolut dunkles Zimmer nötig; auch der schwächste durch den kleinsten Spalt eindringende Lichtstrabl kann das Auge in der Beurteilung des Schirmbildes stark beeinträchtigen. Wer viel durchleuchtet hat, wird diese Tatsache bestätigen können. Es ist deshalb sehr zu empfehlen, im Zimmer nur gedämpftes Lampenlicht zu haben. Und selbst hat sich eine dunkelgelbe Birne sehr gut bewährt. Das Röhrenlicht muss auf jeden Fall für den Beobachter abgedeckt.werden; wir haben dies immer auf die einfachste Weise ausgeführt, indem wir die Röhre mit einem dichten, tief schwarzen Tuch verdeckten. Nur wenn sich Härtegrad der Röhre und Stromintensität nicht entsprachen - wenn der Widerstand in der Röhre dem Strom za gross war —, kamen die Nachteile der Tuchverdeckung zutage; es trat eine reichliche Funkenbildung auf, die ein Durchschlagen der Röhre sehr leicht möglich machte. In diesem Stadium ist die Betriebsunterbrechung der Röhre unbedingt nötig; die mitunter sehr stark erhitzte Röhre muss entweder langsam abgekühlt, oder noch besser gegen eine ausgeruhte ausgetauscht werden.

Bei der längeren Gebrauchsdauer, wie sie eine Thoraxdurchleuchtung erfordert, hat uns eine Umwicklung des Kathodenhalses mit einer feuchten, stark ausgedrückten Mullbinde vortreffliche Dienste geleistet. Das Licht und die Durchdringangsfähigkeit erhielten sich entschieden längere Zeit auf derselben Stufe, und die Betriebsdauer der Röhre war eine grössere. Eine würdige Erklärung dieses Vorganges konnten wir uns nicht verschaffen. 
Eine Einhüllung der Leitungsdrähte in Gummischläuche hat sich uns ferner ebenfalls gut bewährt. Bei einer schon bestehenden oder während des Röhrenganges eintretenden Inkongruenz der Stromstärke und des Härtegrades der Röhre wurde das lästige und die Kranken meist sebr beängstigende Funkenspritzen der Drähte verhütet oder aber wenigstens sehr verringert.

Auf jeden Fall ist es für eine möglichst einwandsfreie Untersuchung erforderlich, das Auge vor Beginn derselben an die Dunkelheit zu adaptieren. Es muss sich erst im Dunkeln ordentlich ausruhen, um ein Maximum der Sehschärfe für das Fluoreszenzlicht zu gewinnen. Sobald das Auge aus dem Tages- oder künstlichen Lichte kommt, besitzt es noch nicht die richtige Empfindlichkeit. Natürlich wird auch die Sehschärfe immer wieder herabgesetzt, sobald gewöhnliches Licht in das Ange gelangt. Es gibt also ein Optimum des Sehens, das immer wieder durch wiederholtes Ausruhen des Auges im Dunkeln zu erlangen ist.

Wie es sich auch bei unseren Untersuchungen herausgestellt hat, leidet die Empfindlichkeit des Fluoreszenzschirmes; unser Schirm hat jetzt nach etwa zwei Jahren stark an Fluoreszenzkraft eingebüsst. Infolgedessen hat es sich als wünschenswert gezeigt, bei den Durchleuchtungen zeitweise den Schirm gegen einen ausgeruhten auszuwechseln, da fernerhin derselbe bei längerer Bestrahlung selbstleuchtend wird, worunter natürlich die Feinheit des Bildes leidet. Es wäre gleich hier noch anzufügen, dass auch die Objekte bei längerer Durchleuchtung für die direkte Bestrablung ungünstiger werden. Die die Röntgenstrablen absorbierenden Medien lassen die sog. Sekundärstrahlen entstehen, die ihrerseits auch eine Fluoreszenz, wenn auch eine schwächere, hervorrufen und dadurch die Bilder verwischen. Dies ist für die Erkennung der Feinheiten ganz besonders ungünstig; darum empfiehlt es sich auch, der immer nur kürzere Zeit in Anspruch nehmenden Röntgenographie vor der Röntgenoskopie den Vorzug zu geben. Die Schirmdurchleuchtung kann bei feineren Untersuchungen mitunter durchaus unzureichend werden; der Schirm ist ja durch die grobkörnige Schichtbeschaffenheit weniger empfindlich als die Platte. Fernerhin muss, wie Holzknecht anführt, die Differenz zweier Lichtintensitäten die sog. Reizschwelle für die Lichtempindlichkeit des Auges überschreiten - der Zuwachs an Intensität ungefähr ${ }^{1 / 30}$ betragen -, um empfunden zu werden. „Das Auge muss also in dem Masse, wie die Intensität Schwankungen unterworfen ist und hier an der Grenze des Wahrnehmbaren leicht unter die Reizschwelle gerät, eine grössere Reihe voneinander folgenden Eindrücken zu einem Bilde summieren; das kann es aber nicht, wohl aber die photographische 
Platte" (Holzknecht). Diese Tatsache haben wir durch Vergleiche von Aufnahmen und Schirmbildern oft bestätigen können. Wir haben auch in den meisten Fällen, bei denen uns die Durchleuchtung in irgend einer Beziehung einen Fingerzeig gab, eine Aufnahme gemacht.

Wir möchten gleich an dieser Stelle eine einfache Methode der Plattenbesichtigung, wie wir sie in unserem Röntgenzimmer anwenden, erwähnen und wegen ihrer Billigkeit und Einfachbeit in Empfehlung bringen. Wir fügen die Platte in einen der Grösse entsprechenden Ausschnitt des schweren Fensterfilzvorhanges. Der Ausschnitt ist an den Rändern innen und aussen mit starken Filzstreifen besetzt, in welche die Platte lichtdicht eingeschoben wird. Das Licht fällt von aussen entweder durch eine matte oder - für einfache Verhältnisse genügend - durch eine mit Filtrierpapier überklebte Fensterscheibe. Durch diese einfache Methode sind wir imstande, die gleichmässig hell erleuchtète Platte im dunklen Röntgenzimmer einer genauen Betrachtung und Prüfung zu unterziehen; wir sind mit diesem Verfahren sehr zufrieden gewesen, zumal wir es auch zu photographischen Reproduktionen (Verkleinerungen) sehr gut benützen konnten.

Wir haben uns ferner bei unseren Durchleuchtungen nie der Blenden bedient; wir wollen aber gleich hier anführen, dass wir uns der grossen Vorteile, die die Blende - vor allen Dingen bei der Schirmanwendung - bietet, wohl bewusst gewesen sind. Uns kam es dagegen sehr darauf an, das ganze Lungenfeld bei genau demselben Licht, in demselben Moment zu übersehen, die verschiedenen Schattierungen auf dem Lungenbilde gleichzeitig miteinander vergleichen zu können. Um sich über eventuelle Nüancen ein genaues Urteil zu bilden, gewährt eine Blende die beste Möglichkeit, falls man nicht gerade eine Aufnahme machen will. Der Schirm leuchtet schon ziemlich intensiv, wenn er nur von schwachen Strahlen getroffen wird; die diffundierten, die sogenannten Sekundärstrahlen, bringen deshalb auf ihn eine verhältnismässig stärkere Wirkung hervor, als auf der Platte, so dass das an sich schon wenig kontrastreiche Bild des Schirmes durch dieselben noch mehr verschleiert wird. In diesem Falle kann man durch Anwendung der Blende, also durch nügglichst grosse Ausschaltung der Sekundärstrahlen, die Kontraste auf dem Schirmbild verstärken. Auch der Vorteil, dass mit der Blende durch Bewegungen des Patienten grössere Partien abgesucht, und so ein genügender Überblick geschaffen werden kann, war uns gegenwärtig. Weil wir aber keine für diese Zwecke handliche Blendeneinrichtung zur Verfügung hatten, zogen wir stets das röntgenographische Verfahren vor. 
Was ferner das Röhrenlicht anbelangt, so haben wir fast immer ein mittelweiches Licht von sattgrüngelber Farbe verwandt. Es hat sich uns gezeigt, dass die Details am deutlichsten bei dem Minimum von Licht erscheinen, das eben noch Wahrnehmungen auf dem Schirm zulässt. Es ist uns aufgefallen, dass Leute mit kräftigem Brustbau bei gleicher Röhrenlichtbeschaffenheit mitunter bessere Bilder gaben, als zarte Individuen mit schwächlichem Thorax, dass bei hochgradiger Adipositas die Verdunkelung des Lungenfeldes soweit ging, dass man unter den besten Bedingungen kein brauchbares Schirmbild des Thorax erhielt. Die massigen Weichteile absorbieren so grosse Intensitätsmengen des Röhrenlichts, dass die Differenz der Weichteilund Knochenschatten im Augenblicksbild des Schirmes leicht so klein wird, dass unser Auge sie nicht mehr wahrnimmt.

Im Anschluss an die Besprechung der Röhrenlichtbeschaffenheit wollen wir eine kurze Darstellung unserer röntgenographischen Methode geben. Wir verwandten zu unseren Aufnahmen weiches sattgrünes Licht mit groben Unterbrechungen (feinster Stift unseres dreiteiligen Wehnelt-Unterbrechers), den Verstärkungsschirm, Schleussners Emulsionsplatten, in der ersten Zeit den Rodinal-, später Hydrochinon- und Glycinentwickler. In den meisten Fällen liessen wir die Kranken während der Aufnahme oberflächlich atmen, in einzelnen Fällen röntgenographierten wir auch im Atmungsstillstand (LevyDorn). Nach den gewonnenen Eindrücken müssen wir sagen, dass wir keine wesentlichen Vorteile von der letzteren Methode gesehen haben. Die Cowl-Guillemontsche Methode, Aufnabmen in bestimmten Atmungsphasen (Inspiration), haben wir nie in Anwendung gebracht. Die Belichtungszeiten dehnten wir auf $3 / 4-1^{3 / 4}-2^{1 / 2}$ Minuten aus; als Massstab dienten uns die Lichtintensität der Röhre und die Beschaffenheit der Thoraxwandung. Zu erwähnen wäre, dass mit mancher Röhre weniger zufriedenstellende röntgenographische Resultate erzielt wurden; es hat uns den Eindruck gemacht, dass dies entschieden an dem Röhrenmaterial gelegen haben muss; denn unter unseren 200 Aufnahmen, die mit den verschiedensten Röhren gemacht wurden, befanden sich Röntgenogramme äusserst scharfer und solche verwaschener Natur. Jedoch können dabei auch verschiedene andere bisher noch unbekannte Faktoren mit im Spiele gewesen sein.

Ganz besonders aufgefallen ist es uns und erscheint deshalb auch erwähnenswert, dass in etwa 10 Fällen der Verstärkungsschirm beim Öffnen der Kassette eine deutliche Fluoreszenz - Schattenumrisse des röntgenographierten Objektes - zeigte, die auch noch längere Zeit - bis 20 Minuten - anhielt. Eine Erklürung für dieses 
uns selten zu Gesicht gekommene Phänomen können wir leider nicht geben.

Nachdem wir so über unsere Röntgenhandwerkzeuge einen Überblick gegeben haben, wollen wir uns im folgenden zur Besprechung des von uns geübten Durchleuchtungsverfahrens wenden. Die Patienten entkleideten ihren Thorax völlig, wurden von dem untersuchenden Arzt zunächst auf Thoraxabnormitäten (Muskeldifferenzen, Wirbelsäulenverkrümmungen usw. geprüft. Es ist ganz besonders darauf hinzuweisen, dass schon geringe, nicht auffallende Grade von Skoliose relativ grosse Veränderungen des Lungenbildes erzeugen. Ferner wurden die Lungenspitzengebiete gründlich auf supra- und infraklavikuläre Drüsen untersucht, da diese leicht Herde in den Lungenspitzen vortäuschen können. Nach diesen Voruntersuchungen brachten wir die Kranken mit der Röntgenröhre in die richtige Stellung. Bei der dorso-ventralen Strahlenrichtung stellten wir die Röhre hinter die Mitte der Interskapularräume, etwa in die Höhe des 4. bis 5. Processus spinosas. Sie muss im Rücken tief genug gestellt werden, so dass sich die Lungenspitzen über den Schatten der Clavicula hinauf projizieren können. Bei der ventro-dorsalen Strahlenrichtung wurde die Röhre, etwa dem. Sterno-Klavikulargelenk entsprechend, eingestellt. Sie muss, um ein gutes hinteres Thoraxbild zu erhalten, so hoch eingestellt werden, damit die Schlüsselbeinschatten unterhalb der Spitzen lokalisiert werden. Im grossen und ganzen ist die Untersuchung in ventro-dorsaler Richtung etwas leichter, da die mehr eben gestaltete Rückenfläche ein bequemeres Absuchen mit dem Schirm zulässt. Bei ventro-dorsaler Strahlenrichtung zeigen die Spitzen die Form eines Winkels, bei dorso-ventraler|Richtung die Form eines Halbkreises. Wenn wir das gesamte Spitzenfeld betrachten wollten, stellten wir die Röhre hinter dem betreffenden Schultergelenk auf, so dass die Spitze mehr in einer schrägen Richtung durchleuchtet wurde. Auf diese Weise wird das Spitzenfeld durch :einen medianen, früher im Mittelschatten verborgenen Lungenanteil vermehrt.

Bei der Durchsuchung der übrigen Lungenpartien haben' wir dann mehrere, in verschiedenen Höhen gelegene $\mathrm{Röhren-}$ stellungen angewandt; wir liessen die Kranken, vor allem sehr dicke Personen tief inspirier en, weil dadurch die unteren Lungenteile heller wurden. Die inspiratorische, überwiegend basale Helligkeitszunahme erklärt sich aus der durch die Zwerchfellsatmung bedingten stärkeren Dehnung und Luftfüllung der basalen Lungenteile. Die Spitze dagegen zeigt ibrer geringeren Ventilation entsprechend keine nennenswerte inspiratorische Aufhellung. Den Skapular- 
schatten verdrängten wir durch Armheben aus dem bellen Lungenfeld. Die Brustdrüsen, ganz besonders stark entwickelte, liessen wir mit einer Bindentour hochbinden oder von den Patientinnen mit der Hand selbst emporheben. Nur so waren, namentlich bei gut genährten Fraven, die Lungenbasen und 7werchfellkuppen zu überseben.

Da mitunter in der einen Durchstrahlungsrichtung mehr, in der anderen weniger, in der einen kleine, in der anderen grosse'Herde zu sehen waren, haben wir jeden Kranken mindestens in zwei Richtungen, in der dorso-ventralen und in der ventro-dorsalen, durchleuchtet. Die Röhre war vom Rücken $20-25 \mathrm{~cm}$, vom Schirm etwa $35-40 \mathrm{~cm}$ entfernt. Bei der Durchleuchtung achteten wir nun auf folgende Punkte:

1. Durchsichtigkeitsveränderungen eines oder beider Spitzengebiete;

2. einzelne Herdschatten;

3. Rippen- und Pleuraschatten;

4. Zwerchfellbewegungen;

5. Veränderungen der Lage der Rippen.

Zunächst sei noch das Durchleuchtungsmaterial einer kurzen Betrachtung unterworfen. Es besteht mit Ausnahme weniger Fälle aus Kranken unserer Frauenstation. Wir haben deshalb das weibliche Material bevorzugt, weil wir der Meinung waren, dass wir bei dem im allgemeinen schwächeren Thoraxbau und bei der auch schwächer ausgebildeten Muskulatur der Frauen bessere und klarere röntgenologische Resultate als bei dem männlichen Krankenmaterial erzielen würden. Wir haben deshalb gerade auch, um uns einzuüben, unsere ersten Untersuchungen an den Frauen angestellt. Nachdem wir uns so gründlich mit dem Verfahren vertraut gemacht und uns immer mehr und mehr von dem Werte der Röntgendurchleuchting überzeugt hatten, gingen wir an eine systematische Untersuchung all unserer Kranken. Wir haben mit Ausnahme einiger Fälle, die wir nur besonderer Merkwürdigkeiten balber in unsere Arbeit aufgenommen haben, die Kranken niemals a usgew ählt, sondern in der Reihenfolge, wie sie in die Anstalt aufgenommene wurden, auch röntgenologisch untersucht und, ohne irgendwelche Fälle auszuschalten, zu unserer Arbeit verwandt.

Über das Krankenmaterial haben wir kurz folgendes zu berichten: 31 Fälle standen im beginnenden Stadium, 173 Fälle waren subchronischer, 114 chronischer Natur. Erbliche Belastung war in 84 Fällen, die wahrscheinliche Infektionsquelle bei 117, Tuberkelbacillen im Auswurf von 41, eine positive Tuberkulinprobe bei 142 Kranken, vorausgegangene Skrofulose bei 50, Stein-, Kalk-, Kohlenstaubinhalation bei 8 , Metallstaubeinatmung bei 30 , Wollstaubein- 
atmung bei 92 , Papierstaubeinatmung bei 36 Kranken zu verzeichnen. Im Alter bis zu 20 Jahren standen 18 Kranke, von 20-30 Jahren 237 , von $30-4053$ Kranke, über 40 Jahre alt waren 12 Kranke.

Von dem angeblichen Beginn der Erkrankung bis zur ersten Durchleuchtung waren verflossen:

$\begin{array}{llll}\text { bis zu 3 Monaten in } & 10 & \text { Fällen } \\ 3-6 \text { Monate } & " & 54 & \\ 6-9 & " & 70 & " \\ 9-12 & " & 37 & " \\ 1-2 \text { Jahre } & " & 69 & " \\ \text { über 2 Jahre } & " & 80 & "\end{array}$

191 Kranke befanden sich in schlechtem, 80 in mittlerem, 49 in gutem Ernährungszustande; in $146^{\circ}$ Fällen war ein schwacher, in 98 ein mittelkräftiger, in 76 ein kräftiger Brustbau zu verzeichnen.

Hinsichtlich der Durchsichtigkeitsveränderungen der Lungenfelder ist $z \mathfrak{u}$ konstatieren, dass sich in den weitaus meisten Fällen die Spitzengebiete gleichmässig, nebelartig, verdunkelt zeigten. Die Schattenbildung ging mitunter über den perkutorisch als krank nachweisbaren Bezirk weit hinaus. Seltener waren zirkumskriptere Schatten, von normal durchlässigen Lungenpartien umgeben, zu sehen. Vielfach zeigte sich eine Verwischung der Umrisse der Rippen und des Schlüsselbeins, was als ein Effekt der im infiltrierten Gewebe entstehenden Sekundärstrahlen anzusehen ist. Auf jeden Fall hat es sich gezeigt, dass die Durchleuchtung keine Aufschlüsse über das Alter der Spitzenerkrankung geben kann. Alte, geschrumpfte Herde, die nur stellenweise floride Prozesse zeigen und klinisch, nicht aber anatomisch, mit dem Bilde der Tuberculosis incipiens zusammenfallen, können dieselben Schattenbildungen wie ganz frische Erkrankungen hervorrufen.

Über den mittleren und unteren Lungenpartien kamen die verschiedenen Schattenbildungen zutage. Herdförmige Infiltrationen erzeugten hellere und dunklere Schatten, sie traten in einer den anatomischen Verhältnissen näher stehenden Weise differenziert vor unser Auge.

$\mathrm{Zu}$ dem, was bis jetzt über den röntgenologischen KK a vernen$\mathrm{n}$ ach w e is bekannt ist, hätten wir auf Grund unserer Untersuchungen nichts besonders Neues hinzuzufügen; nur möchten wir erwähnen, dass bronchiektatische Kavernen in normaler Lungenumgebung dem Auge des Beobachters entgehen können, wenn sie lufthaltig sind. Jedenfalls hat es sich uns deutlich erwiesen, dass die Röntgenstrahlen in vorgeschrittenen Fällen weitaus das beste Übersichtsbild geben, 
eine schärfere Beobachtung des Verlaufes und der Ausbreitung der Erkrankung gestatten.

Ganz besonders wollen wir noch auf die Lungenhiluszeichnung und die sog. Begleitschatten des Herzens hinweisen. Die erstere ist auf dem Leuchtschirm nur unter den günstigsten Bedingungen zu sehen. Bekanntlich sind die Lungen bei älteren Personen, ganz hesonders bei sehr muskulösen und fettreichen Individuen, für die Röntgenstrahlen weniger durchlässig, als bei jüngeren. Ein magerer mit nur geringen Weichteilen bekleideter Thorax lässt ein gutes Bild von der Lungenhiluszeichnung auf dem Schirm entstehen; in jedem anderen Falle ist eine sichtbare Lungenzeichnung als anormal anzusehen. Es ist ja auch leicht verständlich, dass die Hiluszeichnung z. B. bei alten Leuten (Staubablagerungen, Verkalkungen!) und beim substanziellen Emphysem (Bindegewebsbildung) ausgeprägter ist als unter normalen Verhältnissen. Auf der empfindlicheren Platte ist die Lungenzeichnung in den meisten Fällen eine ausgeprägtere als auf dem Leuchtschirm. Das Urteil, ob noch gesund, ob krank, muss mit aller Vorsicht gefällt werden. Im allgemeinen ist die Lungenzeichnung als pathologisch anzusehen, wenn unregelmässige Marmorie. rungen in den peripheren Lungengebieten auftreten.

Wir erachten es als lohnend, noch etwas näher auf die sog. Begleitschatten des Herzens einzugehen. Diese liegen in einiger Entfernung vom rechten Herzrand, links oft hinter dem Herzrand. Anatomisch liegen ihnen die Bronchien und die für die Unterlappen bestimmten stärksten Gefässe zugrunde. Es ist natürlich, dass namentlich bei immer wiederkehrenden Bronchialkatarrhen die Drüsen längs dieser Luftröhrenverzweigungen in einen chronischen Entzündungszustand geraten und so durch vermehrte Lichtabsorption Schattenbilder hervorrufen müssen. Gar häufig haben wir rundliche Schatten in der Lungenhilusgegend und stark ausgesprochene Begleitschatten konstatieren können. In manch zweifelhaftem Falle, bei dem sonst weder klinisch noch röntgenologisch ein Krankbeitsherd in den Lungen nachgewiesen wurde, der den schwerkranken Eindruck des Patienten erklären konnte, glauben wir in diesen Schattenbildungen einen festen Anhaltspunkt $\mathrm{zu}$ haben, wo wir bisher nur auf blosse Vermutungen angewiesen waren. "Jedoch ist wiederum auch grosse Vorsicht bei der Beurteilung dieser Begleitschatten nötig; denn Bronchialknorpel, Bronchiektasien können auch Schattendichten und -hellen geben, und das gesunde Lungengewebe erscheint mitunter durch den Thorax nicht immer in gleichmässiger Helle, sondern oft sogar fleckig." (Gocht.)

Bei zentralen Erkrankungsherden hat sich die Überlegenheit der Röntgenstrahlen den anderen klinischen Untersuchungs- 
methoden gegenüber eklatant gezeigt. Die Herde sind häufig im Zentrum schon viel zahlreicher, als man nach dem klinischen Befunde von vorneherein vermutet hätte. Verhältnismässig kleine zentral gelegene Drüsenpakete, verkalkte Bronchialdrüsen am Lungenhilus liessen sich leicht nachweisen; einige Male haben wir röntgenologisch Infiltrationsherde vor der klinischen Feststellung konstatiert; wir werden später bei Besprechung verschiedener in gewissen Beziehungen markanterer Fälle noch näher darauf eingehen.

Was das Williamssche Symptom - die mangelhafte Abflachung der der erkrankten Spitze entsprechenden Zwerchfellhälfte bei der Inspiration, von Gardiner als sehr wichtiges Frühsymptom der Lungentuberknlose angeführt - anlangt, geht unsere Ansicht nach den gewonnenen Eindrücken dahin, dass es bei Beginn der Tuberkulose als ein unsicheres Phänomen anzuseben ist. Wir haben es in den spärlichen Fällen ganz frischer Tuberkulose unseres Materials niemals deutlich ausgesprochen gefunden, obwohl wir mit grösster Mühe danach gefahndet haben. In der grossen Anzahl unserer tuberkulösen Erkrankungsfälle mehr chronischer Natur haben wir es stets vermisst.

In nur wenigen Fällen haben wir bei sichergestellter tuberkulöser Spitzenerkrankung eine Veränderung der Lage der Rippen konstatieren können, die "sich in einer Abknickung der Rippen nach unten äusserte. Die Rippen werden dadurch sich selbst und der Wirbelsäule genähert" (Hildebrand). Letztere war zuweilen bei ausgesprochenen Erkrankungsfällen nach der erkrankten Seite hin verbogen. „Durch beide Zustände wird der Pleuraraum der betreffenden Seite verkleinert" (Hildebrand). Somit kann quweilen die Röntgendurchleuchtung ein Nachschleppen der erkrankten Thoraxseite und eine sich entwickelnde Schrumpfung früher nachweisen, als man nach der Inspektion des Thorax vermuten würde.

Nach diesen allgemeiner gehaltenen Ausführungen wollen wir zur detaillierten Besprechung unserer Resultate übergehen.

Von den zur Untersuchung gelangten 320 Fällen ergab sich bei 298 ein absolut positives Resultat, während bei 22 Patienten der durch die Röntgen-Durchleuchtung gewonnene Befund mit dem klinischen trotz wiederholter Untersuchungen $n$ icht in volle Übereinstimmung gebracht werden konnte. Auffallend ist, dass ron diesen 22 Fällen mehr als die Hälfte (13) einen schwächlichen, 6 einen mittleren und nur 3 einen wirklich kräftigen Thoraxbau zeigten; die Hälfte (11) befanden sich in schlechtem Ernährungszustande, 8 im mittleren und nur 3 in gutem. 11 führten den Beginn ihrer Erkrankung nur auf mehrere Monate bis höchstens 1 Jahr zurück, während die andere Hälfte schon 1 Jahr lang oder darüber mit 
ihrem Leiden behaftet zu sein angaben. Leider ist es uns nicht gelungen, für diese auch schon von $\mathrm{Kraft}$ und Holzknecht beobachtete Tatsache, dass gerade schwächlich gebaute und mangelhaft genährte Leute mehr negative Resultate bei der Durchleuchtung geben, als kräftig gebaute, in gutem Ernährungszustand befindliche, eine auch nur halbwegs genügende Erklärung zu finden. Wir müssen erwähnen, dass sich bei den meisten dieser Fälle übrigens auch klinisch nur ganz unbedeutende krankhafte Veränderungen feststellen liessen, dass aber bei der grösseren Anzahl derselben die Diagnose Tuberkulose durch positiven Ausfall der Tuberkulinprobe, bei den übrigen durch die Anamnese bezw. durch das Auffinden von Tuberkelbacillen im Auswurf gesichert war. Eigentlich hätte man ja erwarten sollen, dass Leute mit kräftigem Brustbau und gutem Ernährungszustand eher negative Befunde ergeben; denn wie beliannt, entstehen die sogenannten Sekundärstrahlen, die gerade das Röntgenbild undeutlich machen, in dichteren Schichten von Muskel- und Fettgewebe und führen an sich schon leicht zu einer Verdunkelung auch gesunder Lungenspitzengebiete.

Bei unseren positiven Resultaten fällt uns zunächst die Gleichmässigkeit der Schatten verschiedener Intensi$t a ̈ t$ auf, auch in dem grössten Teil der röntgenographisch festgelegten Fälle; hierselbst hatten wir keine Gelegenheit, unsere Befunde auf dem Sektionstische zu kontrollieren; aber wie sonstige pathologische Untersuchungen deutlich zeigen, können wir nicht annehmen, dass wir es in den meisten unserer Fälle mit einer gleichmässigen diffusen Infiltration bestimmter Lungenbezirke $z \mathfrak{u}$ tun hatten - wenn auch vielfach bei frischen Prozessen vermehrter Blut- und Saft- bezw. verminderter Luftgehalt der betr. Spitze die Gleichmässigkeit des Schattens erklären könnte -, weil sonst doch häufig ausgesprochene Narben, eventuell mit Kalkeinlagerungen, gelegentlich von Selktionen gefunden werden. Es ist wenig wabrscheinlich, dass grössere peripher sitzende Herde eine stärkere allgemeine Verdunkelung des Lungenspitzenfeldes bei Durchleuchtung in zwei verschiedenen Richtungen hervorrufen können; denn ein Herd, der an der Vorder- oder Rückseite liegt, müsste entweder bei der einen oder anderen Durchleuchtungsrichtung sich in seiner Grösse und Ausdehnung röntgenoskopisch oder röntgenographisch feststellen lassen.

Hinsichtlich des Prävalierens der Erkrankung der einen oder anderen Spitze fanden wir in $56 \%$ unserer Fälle allein oder vorwiegend die rechte, in $44 \%$ allein oder vorwiegend die linke Spitze von der Erkrankung ergriffen. Wir gelangen damit in Widerspruch mit $C h$. Leroux, der unter $1 弓 4$ Fällen von Kindern im 
Alter von 8-15 Jahren die linke Seite allein $87 \mathrm{mal}$, die rechte dagegen nur $67 \mathrm{mal}$ befallen fand. Wie allgemeine Ansicht der meisten Kliniker und Patbologen, wird vorwiegend die rechte Spitze zunächst von der Tuberkulose ergriffen, und damit würde auch unser Resultat übereinstimmen.

Mit Tuberkulin behandelte Fälle zeigten zuweilen sehr intensive Schattenbildungen in der vorwiegend erkrankten Spitze, wie sie allerdings auch dem während der Behandlung häufiger beobachteten Intensiverwerden der Dämpfung entspricht.

Von Interesse dürften auch jene Fälle sein, in denen die Tuberkulinprobe (höchste Dosis 2,5 mg alten Tuberkulins) negativ ausfiel; dieselben betreffen durchweg Franen. Im ersten Falle handelt es sich um eine $3 / 4$ Jahre vor der Aufnahme in die Anstalt unter typischen Erscheinungen: Stichen, Husten, Auswurf, Nachtschweissen, leichtem Fieber, Gewichtsabnahme erkrankte Näherin; diese Erkrankungserscheinungen verschwanden schon vor der Aufnahme. Klinisch bestand zur Zeit des Eintrittes in die. Anstalt geringe Dämpfung mit vesikobronchialem Atmen über der rechten Spitze ohne Rasselgeräusche. Die Durchleuchtung ergab eine deutliche Beschattung der rechten und eine schwache der linken Spitze. Ein Jahr nach der Entlassung wurde Patientin zur Kontrolle wieder untersucht; das Allgemeinbefinden war gut, desgleichen der Ernährungszustand; Husten, Auswurf oder sonstige Krankheitserscheinungen hatten sich nicht wieder eingestellt; Patientin hatte andauernd gearbeitet. Objektiv fand sich derselbe Befund wie bei der Aufnahme; lediglich Erscheinungen eines abgelaufenen Prozesses über der rechten Lungenspitze.

Eine zweite Patientin, ebenfalls Näherin, ist 2 Jahre vor der Aufnahme unter denselben Erscheinungen, wie die eben Erwähnte, erkrankt. Bei der Aufnahme bestand etwas Hüsteln und schleimiger Auswurf. Klinisch waren beide Spitzen leicht gedämpft, auf der äussersten rechten Spitze vesikobronchiales Atmen, niemals Rasselgeräusche. Bei der Durchleuchtung zeigte sich deutliche Beschattung beider Spitzen, rechts mehr als links.

Ganz ähnliches Verhalten zeigten die übrigen Fälle, bei denen die Tuberkulinprobe negativ ausfiel und bei denen hieraus, wie auch aus Anamnese und klinischem Befunde, darauf geschlossen werden muss, dass es sich um einen abgelaufenen Prozess in den Lungen, um inaktive Tuberkulose handelt.

Aus dieser T'atsache müssen wir folgern, dass es in manchen Fällen direkt unmöglich ist, mittelst der Röntgenstrahlen zu entscheiden, ob es sich bei klinisch von der Norm abweichenden Befunden über den Lungenspitzen um frische, noch bestehende $\mathrm{Er}$ - 
krankungen, oder um abgelaufene Prozesse mit Narbenbildungen (Bindegewebe, Kalkeinlagerungen) handelt. Anzunehmen wäre natürlich, dass eine allgemeine frische Infiltration in den Lungenspitzen eine beträchtlichere Verdunkelung des Lungenspitzenfeldes im Schirm hervorrufen müsste, als eine Narbenbildung, die in ihrer Gesamtheit noch nicht so viel Röntgenstrahlen absorbieren kann, wie eine frisch infiltrierte Lungenspitze mit vermehrtem Flüssigkeits- und vermindertem Luftgehalt. Es ist mitunter sehr schwierig, da wirklich kritisch vorzugehen, denn wie es unsere zahlreichen Durchleucbtungen gezeigt haben, ist es häufig der Fall gewesen, dass bei Lungenspitzenerkrankungen das Schirmbild in der einen Durchleuchtungsrichtung dem Auge heller erschien als in der anderen. Frische Herde in den Lungenspitzen können natürlich zentral oder peripher sitzen, genau so gut wie Schwielen- oder Narbenbildungen. Diese Lokalisation der verschiedenen Stadien der Lungenerkrankung ist natürlich äusserst wichtig für das Zustandekommen des Spitzenschattenfeldes auf dem Leuchtschirm. Sich allein auf Grund der klinischen Untersuchung ein richtiges Urteil über den betreffenden Zustand der Lungenerkrankung (Sitz, Ausbreitung und Stadium) zu bilden, ist ja, wie allgemein bekannt, zuweilen durchaus nicht leicht, und wir müssen hier gleich anfügen, dass uns auch die Röntgenstrahlen bei Beurteilung dieser Verhältnisse sehr oft im Stich gelassen haben. Bei mehreren (21) unserer Fälle fanden sich bei der Durchleuchtung sowohl der Ausdehnung als der Intensität nach ausgebreitetere bezw. intensivere Schatten, als nach dem klinischen Bild zu vermuten war. Bei einer grossen Anzahl dieser Fälle zeigte der weitere Verlauf teils noch während der Beobachtung in der Anstalt, teils aber auch erst gelegentlich der etwa ein Jahr nach der Entlassung stattgefundenen Kontrolluntersuchung der Kranken, dass spätere klinische Untersuchungen das Resultat der Durchleuchtung bestätigten. In diesen Fällen handelte es sich rorwiegend um Individuen mit schwächlichem Brustbau und schlechtem Ernährungszustand. Die Hälfte (11) von diesen Fällen führte den Beginn ihrer Erkrankung auf weniger als ein Jahr zurück, die andere Hälfte (10) zum Teil auf ein Jahr, zum Teil aber auf sehr lange Zeit (3, 7, 25 Jahre). Diese Befunde erschienen uns befremdend. Die Erkrankung der betroffenen Lungenbezirke musste in den erwähnten Fällen schon ziemlich weit vorgeschritten sein, um sie durch Schattenbildung für das menschliche Auge deutlich bemerkbar zu machen; klinisch konnte die Erkrankung nicht als eine so schwere angenommen werden, aber das Allgemeinbefinden und Aussehen der Patienten, die Beschaffenheit des Auswurfs, der weitere Verlauf der Erkrankung und das Ergebnis der späteren Nachunter- 
suchungen sprachen deutlich dafür, dass es sich um eine wohl schon zur Zeit der Aufnahme bezw. Durchleuchtung weiter vorgeschrittene Erkrankung handeln musste, zu deren richtigen Würdigung eben die klinischen Untersuchungsmethoden der Perkussion und Auskultation nicht ausreichten. Gerade bei diesen Fällen haben wir den Eindruck gewonnen, dass die Röntgenstrahlen der klinischen Diagnostik zuweilen überlegen sind.

Auf Grund mehrerer typischer, markanterer Fälle scheint uns, dass die röntgenologische Diagnostik den pathologisch-anatomischen Verhältnissen besser gerecht wird, als die beiden sonst meist geübten Untersuchungsmethoden der Perkussion und Auskultation; namentlich was gerade die Tiefen- und Breitenausdehnung der Erkrankungsherde anlangt, ist es mitunter sehr schwer, mittelst der älteren Untersuchungsmethoden eine genauere Begrenzung der tiefer gelegenen Herde zu erreichen. Dagegen bietet die Durchleuchtung des Thorax in den verschiedensten Durchmessern und ganz besonders die Beiziehung orthodiagraphischer Bestimmungen die grösste Möglichkeit, sich ein ziemlich genau zutreffendes Bild von der Ausdehnung der Krankheitsherde $\mathrm{zu}$ verschaffen. Namentlich bei dicken Pleuraschwarten, bei denen es manchmal sehr schwierig ist, das Vorhandensein eines Exsudates auszuschliessen bezw. festzustellen, kann das Röntgenverfahren sehr wertvolle Aufschlüsse geben. Bei Besprechung der besonders interessanten Fälle werden wir hierauf noch zurückkommen.

Gerade das zum grossen Teil sehr grazil gebaute und schlecht genälurte Material der Franenabteilung hat es uns deutlich gezeigt, dass die Bronchial- und Gefässverzweigungen in Höhe der dritten und vierten Rippe, also dem Lungenhilus entsprechend, mitunter, scharf gezeichnet, sichtbar sind. Nach dem Alter der Patienten, dem ganzen klinischen Befund und dem sonstigen Eindruck, den die betreffenden Kranken machten, war auf keinen Fall anzunehmen, dass gröbere Veränderungen in den Bronchialverzweigungen oder Gefässen, oder auch ausgedehntere peribronchitische Herde vorlagen. Anders bei Männern, wo es sich um andauernde Inbalation von Kohle-, Kalk-, Stein- oder Metallstaub handelte; von 12 solcher Fälle, die im Alter zwischen 23 und 56 Jahren standen, fanden sich bei 10 deutlich ausgesprochene Begleitschatten des Herzens und Schatten in der Gegend des Lungenhilus. In einem Fall, der eine exsudative Pleuritis links zeigte; war natürlich auf der linken Seite infolge des starken Exsudatschattens von einer ausgesprochenen Bronchialbaumzeichnung nichts zu sehen. In einem andern Fall mit frischer trockener Pleuritis fand sich bis auf eine leichte diffuse Schattierung der entsprechenden Unterlappengegend nichts von ver- 
minderter Durchstrahlungsfähigkeit. Ein dritter Fall war in dieser Beziehung vollständig negativ. Bei all diesen Patienten, deren Durchleuchtung eine grössere Ausbreitung der Lungenerkrankung zeigte, als die klinische Untersuchung, und die ausserdem einen schwerkranken Eindruck machten, bestand eine deutliche Schattenzeichnung, den Bronchial- und Gefässverzweigungen entsprechend.

Zur Erklärung des deutlicheren Hervortretens der Bronchialverzweigung im Schirmbild möchten wir folgendes anführen: Die eingeatmeten Staubpartikelchen werden in den Lympbbahnen der Bronchien und des Lungengewebes bis $z \mathfrak{u}$ den bronchialen und mediastinalen Lymphdrüsen befördert und gelangen dort, wie auch im interalveolaren und subpleuralen Bindegewebe zur Ablagerung. Gerade die häufiger gefundene stark ausgeprägte Schattierung zwischen 3. und 4. Rippe lässt deutlich darauf schliessen, dass der angesammelte Staub in den dort befindlichen Lymphdrüsen einen chronisch entzündlichen Zustand hervorruft. Die nächsten Folgen der Staubinhalation sind Bronchialkatarrhe, die sich anfangs seltener, später immer häufiger wiederholen und dann eine immer längere Dauer annehmen; zuweilen treten dann, namentlich wenn die Katarrhe einen heftigeren Charakter zeigen, bronchopneumonische Entzündungen auf, an denen besonders das interstitielle Bindegewebe beteiligt ist. Schwielenbildungen und Schrumpfung in demselben sind dann der gewöhnliche Endzustand.

Von diesen bronchopneumonischen Prozessen und deren Folgezuständen (Atelektasien und Bronchiektasien) werden von der Lunge besonders die unteren Partien bevorzugt; in den Lungenspitzen dagegen endigen die chronisch-katarrhalischen Zustände meist mit mehr oder weniger ausgedehnten geschrumpften Indurationen, die, wenn auch seltener, gleichfalls erweiterte Bronchien einschliessen können.

Oft ist uns bei der Durchleuchtung, namentlich. unserer weiblichen Kranken, aufgefallen, dass bei sonst wenig ausgesprochenen Spitzenschatten sich deutliche "Begleitschatten des, Herzens" fanden. Wir suchten u. a. gerade die bei Phthisikern so hänfig vorhandenen bestimmt lokalisierten Brustschmerzen (zwischen den Schulterblättern oder mitten vorne auf der Brust, dem Sternum entsprechend) mit diesen Schatten und namentlich mit den marmorierten Schatten zwischen 3. und 4. Rippe, also dem Lungenhilus entsprechend, in Einklang zu bringen. Es ist uns jedoch nicht gelungen, irgend eine greifbare Übereinstimmung herbeizuführen.

In einzelnen Fällen fand sich ein auffallend kleines Herz, das mit der.Spitze dem Zwerchfell aufruhte und sich bei tiefer Inspiration deutlich von der linken Zwerchfellhälfte abhob. Diese Kranken klagten durchweg über häufige Herzpalpitationen; klinisch war in keinem 
dieser Fälle ein pathologischer Befund zu erheben. Bei 12,5\% derjenigen Patienten, welche besonders deutlich gezeichnete Begleitschatten des Herzens zeigten, fand sich eine ausgesprochene Pulsbeschleunigung, auch in der Ruhe; hiervon war nur eine Patientin mit einem deutlichen Herzfehler, eine weitere mit einem auffallend kleinen Herzen. Bei den übrigen konnte eine klinische Ursache für die anhaltende Pulsbeschleunigung, die von manchen Autoren als prognostisch ungünstiges Zeichen bei beginnender Langentuberkulose gedentet wird, nicht gefunden werden; insbesondere lag bei diesen anderen gegenüber keine auffallende Nervosität vor. Die Möglichkeit, dass durch vergrösserte Bronchialdrüsen ein Druck auf den Vagus und dadurch eine Pulsbeschleunigung hervorgerufen wird, ist nicht von der Hand zu weisen.

Die erwähnten Begleitschatten fanden wir auch fast stets bei Fällen von chronischer Bronchitis und Bronchoblennorrhöe. Eine Ausnalıme bildet nur folgender Fall: Eine 20jährige Näherin zeigte sehr reichlichen eiterigen Auswurf bei geringen klinischen Erscheinungen und gutem Ernährungszustand, dabei gutes Allgemeinbefinden; objektiv bestand nur rauhes Atmen über beiden Unterlappen, geringe Dämpfung über der einen Spitze bei vesikobronchialem Atmen. Tuberkulinprobe positiv. Bei der Durchleuchtung fand sich nur mässig verminderte Durchlässigkeit der erkrankten Spitzen, In einem weiteren Fall dagegen, der einen Mann in vorgerücktem Alter mit reichlichen klinischen Erscheinungen einer chronischen Bronchitis mit konsekutivem Emphysem und Asthma betraf, liessen sich die Begleitschatten des Herzens deutlich nachweisen; ganz besonders auffallend waren hier anf der rechten Seite, wo gewöhnlich die Begleitschatten freier dalagen, als links, nach der $\mathrm{Z}$ werchfellkuppel hinziehende fadenförmig verzweigte Schatten. In dem letzteren Fall ist es auf Grund des klinischen Befundes anzunehmen, dass schon gröbere Veränderungen in dem Bronchialbaum vorlagen als in dem ersteren Falle. Die Gegenüberstellung dieser beiden Krankheitsbilder weist daraufhin, dass die Röntgenstrablen mitunter in den zweifelhaften Fällen von chronischer Bronchitis den klinischen Untersuchungsmethoden auch nicht überlegen sind.

Wir glauben, dass es namentlich bei Leuten in vorgerückterem Alter, die andauernder Staubinhalation ausgesetzt waren, und bei denen sich schon gröbere Veränderungen in den Bronchialverzweigungen annehmen liessen, bedeutend schwerer ist, durch die RöntgendurchFeuchtung ein verwertbares Resultat zu erreichen, als bei jugendlichen Individuen, bei denen eine Verkalkung der Bronchialknorpel oder sonstige Prozesse, die eine deutliche Schattenbildung hervorrufen könnten, mit an Sicherheit grenzender Wahrscheinlichkeit auszuschliessen sind. 
Die bei Lungentuberkulosen zuweilen za beobachtenden chronischen trockenen Bronchitiden, die häufig nur durch Digitalis oder doch nur durch Krärtigung des Herzens (ohne dass am Herzen nachweisbare Störungen vorhanden wären) zu bekämpfen sind, gaben niemals ein positives Röntgenbild.

Zwei Fälle von Bronchiektasien und Residuen von Unterlappen-Pneumonien ergaben bei der Durchleuchtung verschiedene Resultate: eine Patientin, bei der die Diagnose Lungentuberkulose fraglich war, litt klinisch an ausgedehnten Bronchiektasien mit reichlichem fötiden, eiterigen, zuweilen auch sanguinolenten Auswurf. Bei der Durchleuchtung fanden sich diffuse Schatten in beiden Unterlappen. Bei einer anderen Patientin bei ähnlichem klinischen Bilde, bei der es sich nach der Anamnese um Residuen beiderseitiger Unterlappenpneumonie handelte, fanden sich bei der Durchleuchtung nur starke Begleitschatten des Herzens; die Unterlappen dagegen liessen die Röntgenstrahlen, trotz der deutlichen klinischen Erscheinungen vollständig normal durch. Bei zwei männlichen Patienten, die in beiden Unterlappen Reste von früher überstandenen Pneumonien aufwiesen, bestand starke Schattenbildung den erkrankten Partien entsprechend, in einem Falle ausserdem auch noch ein deutlich ausgesprochener Begleitschatten des Herzens rechts. Dass diese Schatten jedenfalls nicht ausschliesslich durch pleuritische Schatten hervorgerufen sein konnten, geht daraus hervor, dass wir in anderen Fällen von pleuritischen Schwarten (Residuen früher ïberstandener exsudativer Pleuritis), die sich klinisch durch geringe Dämpfung und abgeschwächtes, sonst nicht verändertes Atmungsgeräusch kundgaben, in der Regel keine Schattenbildung sahen; jedenfalls beobachteten wir niemals in derartigen Fällen eine Schattenbildung von solcher Intensität, dass sie der eben erwähnten bei Residuen früherer Pneumonien auch nur annähernd gleichkam. Auch sprachen die klinischen Erscheinungen in diesen Fällen stets für pathologische Veränderungen im Lungengewebe, nicht am Rippenfell.

Bei Pleuritiden war das Resultat meist negativ. Nur in frischen Fällen (exsudative Form) zeigte sich ein starker Schatten, den betreffenden Unterlappen entsprechend. Bei Fällen von einfacher trockener Pleuritis konnte niemals - auch nicht bei Durchleuchtung in verschiedener Richtung -, ein deutlicher Schatten beobachtet werden; auch ein Nachschleppen, eine verminderte Exkursionsfähigkeit der entsprechenden Zwerchfellseite war nicht deutlich zu sehen, obwohl wir die Röhre richtig auf die $Z$ werchfellhöhe einstellten. Die Senkung der Rippen dagegen, wie die Verschmälerung der Inter- 
kostalräume bei exsudativer Pleuritis konnten wir in einigen Fällen deutlich nachweisen.

In folgendem mögen noch mehrere von denjenigen Fällen kurz besprochen werden, die bei der Durchleuchtung bezw. bei Aufnahmen Besonderheiten boten.

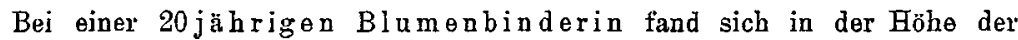
3.-4. Rippe ein kirschkerngrusser, scharf umschriebener Schatten; ein diesem Schatten klinisch entsprechender Befund konnte nicht konstatiert werden. Wir vermuten, dass es sich hier um eine isolierte verkalkte Drüse, vielleicht auch um einen abgeheilten Herd mit Kalkeinlagerung handelt. Der Lungenbefund der betreffenden Kranken zeigte sich ein Jahr nach der Entlassung gebessert.

Eine $22 \mathrm{jahhrige} \mathrm{Näh}$ erin, seit $1^{1} / / \mathrm{Jahr}$ lungenleidend, wies bei der Aufnahme nur geringe Dämpfung über der rechten Spitze mit vesikobronchialem Atmen auf; niemals Rassel- oder sonstige Nebengeräusche; beständig subfebrile bis febrile Temperaturen. Die Durchleuchtung ergab bei der Aufnahme ein vollständig negatives Resultat, ausser dentlich ausgesprochenen Begleitschatten des Herzens. Da die Kranke keine Fortschritte machte, wurde sie mit Neutuberkulin, zunächst T-R, später Bacillenemulsion behandelt, jedoch obne greifbaren Érfolg. Klinisch bildete sich im Laufe dieser Behandlung eine deutliche Dämpfung über der rechten Spitze aus, ohne sonstigen objektiven Befund. Die Durchleuchtung, welche mehrfach vorgenommen wurde, zeigte stets intensiver werdende Beschattung, die bald auf eine grössere Ausbreitung des Krankheitsprozesses schliessen liess, als klinisch festgestellt werden konnte. Die etwa $1 / 2 \mathrm{Jahr}$ nach der Entlassung vorgenommene Nachuntersuchung deckte eine wesentliche Verschlechterung auf: ausgebreitete Infiltration mit absoluter Dämpfung über dem ganzen rechten Oberlappen bei andauernd hohem Fieber (39,0 und zuweilen darüber); auch jetzt noch keine hasselgeräusche, kein Husten, kein Auswurf.

Ein seit ${ }^{3 / 4}$ Jahren krankes $21 \mathrm{jähriges} \mathrm{K}$ inderfräulein zeigte beständig subfebrile Temperaturen. Befund: geringe Dampfong über beiden Spitzen, daselbst ranhes Atmen, keine Rasselgeränsche; niemals Husten oder Auswurf; beschleunigte Herztätigkeit. Tuberkulinprobe vor der Aufnahme positiv. Die Durchleuchtung ergibt starke Beschattung der oberen Hälfte beider Oberlappen, ferner ausgesprochene Schatten in der Lungenhilusgegend. Die ein Jahr nach der Entlassung vorgenommene klinische Untersuchung ergab Verschlimmerung der Lungenerkrankung bei schlechtem Allgemeinbefinden, Nachtschweissen, Gewichtsabnahme und dauernden Temperatursteigerungen.

Eine 19 jährige Arbeiterin, seit mehreren Jabren lungenkrank, zeigt bei der Aufnahme geringe Dämpfung über der rechten Spitze, daselbst vesikobronchiales Atmen und vereinzelte Rasselgeräusche. Die wenige Tage später vorgenommene Durchleuchtung ergibt in der Höhe der 2. und 3. Rippe rechts einen deutlich umschriebenen rundlichen Schatten. Bei weiterer klinischer Beobachtung stellte sich allmählich eine deutliche Dämpfung auch unterhalb der Clavikula und Spina scapulae ein, vesikobronchiales Atmen bis zur 3. Rippe, das mit der Zeit fast rein bronchialen Charakter annahm. Nach etwa neun Wochen wurde die Dämpfung in dem angegebenen Bezirk fast absolut, auch fanden sich bald im Auswurf Tuberkelbacillen, die anfangs trotz häufiger Untersuchungen, Zentrifugierens etc. nicht auffindbar waren. Bei der ein Jahr später vorgenommenen Kontrolluntersuchung zeigte sich eine erhebliche Verschlimmerung der Lungenerkrankung trotz leidlichen Allgemeinbefindens und guten Firnährungszustandes. 
Eine 24 jährige Falzerin, seit einem halben Jahre lungenkrank, zeigt bei der Aufnahme über der rechten Spitze Dämpfung, vesikobronchiales Atmen und vereinzelte Rasselgeräusche. Bei der Durchleuchtung zeigt sich die obere Hälfte des rechten Oberlappens stark beschattet; der weitere klinische Verlauf entspricht diesem Befunde: allmählich breiteten sich die Rasselgeräusche, die bald einen feuchten Charakter annahmen, bis zur 3. Rippe bezw. Mitte der Skapula aus, im Auswurf erschienen Tuberkelbacillen. Da sich die Kranke fortwährend verschlechterte, musste sie als aussichtslos entlassen werden.

Ein $45 \mathrm{jähriger}$, sehr blutarmer Tischler erkrankte $1 / 2 \mathrm{Jahr}$ vor der Aufnahme mit Hüsteln, etwas Auswurf, linksseitigen Brustschmerzen, Nachtschweissen, Appetitlosigkeit und Heiserkeit. Klinisch bestand mittlere Schallverkürzung bei vesikulärem Atmen über der linken Spitze obne Rasselgeräusche; später rauhes Atmen; die rechte Spitze zejgte ganz minimale Schallverkürzung, rauhes Atmen mit verlängertem Exspirium. A m rechten Stimmband bestand ein tuberkulöses Geschwür. Im Auswurf konnten Tuberkelbacillen nicht nachgewiesen werden. Die Durchleuchtung ergab eine ziemlich beträchtliche Beschattung des rechten, dagegen eine schwache, nur bei ventrodorsaler Strahlenrichtung sichtbare Beschattung des linken Lungenspitzenfeldes. Beiderseits deutliche Begleitschatten des Herzens. Der Patient machte während der langen Beobachtungszeit stets einen schwerer kranken Eindruck, als dem klinischen Befunde entsprach. Ein anderes organisches Leiden konnte mit Sicherheit ausgescblossen werden. Die nach einem Jahre stattgefundene Nachuntersuchung ergab eine ziemlich beträchtliche Verschlechterung des Lungenleidens: Intiltration der oberen Hälfte des rechten Oberlappens mit mässig reichlichem trockenen Rasseln; über der linken Spitze leichte Schallverkürzung mit abgeschwächtem rauhen Atmen.

Ein seit einem Jahre kranker $24 \mathrm{Jahre}$ alter Schneider, der als Kind an skrofulöser Augenentzündung gelitten haben will, zeigte beim Eintritt in die Anstalt leichte Schallverkürzung und vereinzeltes Knacken über beiden Spitzen, links abgeschwächtes Atmen, im linken Unterlappen Giemen. Tuberkulinprobe positiv. Die Durchleucbtung ergab deutliche Beschattung beider Spitzen und einen starken Schatten rechts vom Herzen, sowie faustgrosse Schatten, dem Lungenhilus entsprechend. Drei Wochen später war die Dämpfung über der rechten Spitze deutlicher, bei verlängertem Exspirium und knackenden Geräuschen daselbst; links unten vesikobronchiales Atmen mit veriängertem Exspirium. Die Durchleuchtung ergab dasselbe Resultat wie bei der Aufnahme. Nach weiteren drei Wochen zeigte sich die Dämpfung wiederum stärker als vorher, auch trat über der rechten Spitze vesikobronchiales Atmen auf; links unten deutliches Reiben, kein Exsudat. Die Dämpfung wurde dann rechts immer dichter, die Atmung nahm einen bronchovesikulären Charakter an, auch traten deutliche Rasselgeräusche und im Auswurf Tuberkelbacillen auf. Das Atmungsgeräusch über der linken Spitze wurde vesikobronchial. Nach einem halben Jahre deutliches Rasseln in Höhe der rechten Spina scapulae, desgleichen links hinten oben. Die spätere Kontrolluntersuchung ergab eine erhebliche Verschlimmerung des Lungenleidens.

Ein erst seit einem Vierteljahre kranker $K_{0}$ nt oris t, $28 \mathrm{Jah}$ re alt, machte einen schwerkranken Eindruck bei geringem objektiven Befunde. Bei der Aufnahme bestand leichte Schallverkürzung und rauhes abgeschwächtes Atmen mit verlängertem Exspirium über der linken Spitze, leichte Schallverkürzung und abgeschwächtes Atmen links in der Seite, verlüngertes Exspirium und rauhes Atmen über der rechten Spitze. Tuberkulinprobe positiv. Die Durchleuchtung 
ergab schon bei der Aufnahme eine deutliche Beschattung der rechten, eine mässige der linken Spitze, sowie einen deutlichen Schatten neben der rechten Herzgrenze. Der weitere klinische Verlauf bestätigte das Resultat der Röntgen-Durchleuchtung. Bei der Nachuntersuchung war der klinische Befund unverändert wie bei der Entlassung.

Ähnlich verhielt sich ein seit $1^{1 / 4}$ Jahren kranker $50 \mathrm{Jahre}$ alter Cigarettenarbeiter. Bei der Aufnahme bestand leichte Schallverkürzung über beiden Spitzen, rechts mehr als links; rechts ausserdem rauhes Atmen, keine Rasselgeräusche. Tuberkulinprobe positiv. Die Durchleuchtung zeigte deutJiche Beschattung der oberen Hälfte des rechten Oberlappens, mittlere der linken Spitze und diffuse Schatten im Mittel- und Unterlappen. Auch hier zeigte die weitere Beobachtung, dass es sich tatsächlich um eine viel vorgeschrittenere $\mathrm{Er}$ krankung handelte, als der klinische Befund bei der Aufnahme annehmen liess. Bei der Nachuntersuchung war'der Befund gegenüber dem bei der Entlassung erhobenen unverändert.

Ein seit drei Jahren kranker Maurer, $40 \mathrm{Jahrealt}$, gibt an, früher viel an skrofulösen Drüsenschwellungen gelitten zu haben. Bei der Aufnabme bestand mässige Dämpfung über der rechten Hälfte des rechten Oberlappens, daselbst abgeschwächtes vesikobronchiales Atmen, Giemen und mässig reichliches Rasseln; über der linken Spitze lejchte Schallverkürzung. Die Durchleuchtung zeigte deutliche Beschattung der oberen Hälfte des rechten Oberlappens, mittlere der linken Spitze, sehr deutliche Zeichnung des Bronchialbaumes. Bei der Nachuntersuchung zeigte sich die Lungenerkrankung verseblechtert.

Wir sind der Ansicht, dass bei den bisher angeführten und einer noch grösseren Anzahl ähnlich gelagerter Fälle die Röntgenstrahlen ihre Überlegenheit gegenüber den anderen klinischen Untersuchungsmethoden gezeigt haben. Die ziemlich dichte oder ausgebreitetere Schattenbildung bei verhältrismässig geringem klinischen Befunde sprach entschieden für eine weiter ausgebreitete Erkrankung, als man mittelst Perkussion und Auskultation nachweisen konnte. Die etwa ein Jahr nach der Entlassung stattgefundenen Kontrolluntersuchungen, teilweise aber auch schon die weiteren Beobachtungen in der Anstalt, bestätigten nachträglich das Resultat der Röntgendurchleuchtung.

Von Interesse sind auch noch folgende Fälle:

Bei einem $21 \mathrm{~J}$ ahre alten, angeblich seit fünf Jahren kranken Schreiber fand sich eine $f$ ische Erkrankung über der rechten, eine anscheinend ältere über der linken Spitze; leichte Schallverkürzung mit rauhem Atmen und ver]ängertem Exspirium, sowie vereinzeltem Knacken über der rechten Spitze, mittlere Dämpfung, abgeschwächtes vesikobronchiales Atmen mit vereinzelten Rasselgeräuschen über der linken Spitze. Die Durchleuchtung zeigte deutliche Beschattung der oberen Hälfte des rechten Oberlappens, mittlere Beschattung der linken Spitze und deutliche Zeichnung des Bronchialbaumes. Hier gab also die offenbar frischere Infiltration der rechten Spitze eine intensivere Beschattung als die jedenfalls seit rielen Jahren erkrankte linke Spitze. Befund bei der Nachuntersuchung nach einem Jahre: Unverändert wie bei der Lintlassung.

Ein $54 \mathrm{Jahre}$ alter Maschinist, der seit 25 Jahren krank sein will, zeigte leichte Schallverkürzung über der linken Spitze bei abgeschwächtem Atmen, 
rechts verlängertes Exspirium; im Auswurf Tuberkelbacillen vorhanden. Die Durchleuchtung ergab links starke, rechts weniger ausgesprochene Schattenbildung; in der linken Lunge ausserdem auffallend deutliche Bronchialbaumzeichnung; rechts derber Begleitschatten des Herzens. Patient besass einen äusserst entwickelten Knochenbau, sehr kräftige Muskulatur bei schlecht entwickeltem Fettpolster. Es bandelte sich bei ihm um Arteriosklerose; die ausserordentlich deutliche Zeichnung des Bronchialbaumes dürfte wobl in diesem Falle auf Kalk. ablagerungen in den Knorpeln der grösseren Bronchien, auf Veränderungen der lungengefässe zurückzuführen sein.

Ein $44 \mathrm{j}$ ah higer Mechanike r hatte vor zehn Jahren eite leichte trockene Pleuritis und leidet seit $1 / 2 \mathrm{Jahr}$ an exsudativer Pleuritis rechts und beiderseitiger ausgesprochener Spitzenerkrankung. Tuberkelbacillen waren niemals nachweisbar, anch war Fieber während seines Anfenthaltes in der Heilstätte nie vorhanden; Dämpfung über beiden Spitzen, rechts mehr als links, abgeschwächtes vesikobronchiales Atmen mit verlängertem Exspirium; rechte Spitze Knacken, links mässig reichliches Rasseln; rechts vorne 4.-6. Rippe, hinten 6. bis 10. Processus spinosus, stark ausgesprochene Dämpfung und sehr stark abgeschwächtes Atmen; Pektoralfremitus aufgehoben. Nach acht Tagen Entleerung von $1300 \mathrm{ccm}$ serösen Exsudates; danach klinischer Befund unverändert. Vier Wochen später nochmals Entleerung von $1025 \mathrm{ccm}$ serösen Exsudates. Klinischer Befund: Rechts vorne Dämpfung ab 5. Rippe noch stark, hinten Befund unveründert. Nach weiteren zehn Tagen rechts hinten bei tiefer Atmung Reiben zu hören, Stimmfremitus stark abgesehwächt, aber eben noch fühlbar, und ungefähr nach einer weiteren Woche noch derselbe klinische Befund; Stimmfremitus eben noch angedeutet.

Es war in diesem Falle - wegen der dicken Pleuraschwarten - besonders schwer, sich mittelst der Perkussion und Auskultation über den Grad der Erkrankung der unteren rechten Lungenhälfte, über eine etwaige Fliussigkeitsansammlung, bezüglich deren Ausdehnung Aufschluss zu verschaffen. Die Röntgendurchleuchtung zeigte uns: Vor der Punktion einen dichten Schatten über der rechten unteren Lungenhälfte (ventrodorsale Richtung); in diesem Schattengebiet war ein mehr längliches, in seinen Umrissen verwaschenes Schattenfeld sichtbar, das schräg von der Mitte der Skapula nach der Mitte der rechten Zwerchfellhälfte verlief. Nach der Punlstion blieb die Beschattung der unteren Lungenhälfte; sie wurde nach der Gegend des Lungenhilus zu lichter und war daselbst - Höhe des 5.-6. Brustwirbels - scharf konvex nach oben abgegrenzt. Dicht über dem Zwerchfell war sie ziemlich stark ausgesprochen und endete lateralwärts, von dem oben erwähnten länglichen, bandförmigen Schattenfeld scharf begrenzt. Zwischen letzterem und der äusseren Thoraxwand, die eine nur wenig auffallende Śchattenbildung erzeugte, befand sich ein langgestrecktes, bis zur Mitte der Skapula reichendes spitzwinkeliges Dreieck, das im Vergleich zum gesunden, linksseitigen Lungenfeld bedeutend lichter war. Die das Dreieck durchziehenden Rippen traten sehr deutlich und scharf konturiert hervor. Sollte es 
sich hier um einen lufthaltigen Raum handeln? Nach dem Resultat der Röntgenuntersuchung konnte man dies annehmen. Jedoch waren mit den klinischen Untersuchungsmitteln absolut keine Anzeichen dafür aufzufinden.

Da innerhalb weiterer 14 Tage die klinische Untersuchung über etwaige Veränderungen keinen Aufschluss gab, machten wir noch eine zweite Aufnahme in ventrodorsaler Richtung, die uns wieder ein anderes Bild zeigte: die Schattenbildung entsprach in ihrer Ausdehnung dem ersťen Röntgenbefunde; von dem spitzwinkeligen hellen Dreieck war dagegen nichts zu sehen. Der klinische Befund änderte sich in der folgenden Zeit äusserst wenig; nach drei Wochen ergab dagegen eine Probepunktion wieder seröses Exsudat; mit der anschliessenden Punktion wurden $700 \mathrm{ccm}$ Flüssigkeit entleert. Das sofort nach der Punktion aufgenommene Röntgenogramm zeigte dasselbe Bild, wie das nach der 2. Punktion angefertigte. Das lichthelle spitzwinkelige Dreieck war wieder in aller Schärfe zu sehen. Es drängte sich uns der Gedanke auf, dass hier vielleicht ein HydroPneumothorax vorliegen konnte; klinisch liess sich aber für das Vorhandensein eines solchen ein vollgültiger Beweis nicht erbringen. Auf jeden Fall weisen uns aber die Röntgenstrahlen auf Veränderungen im Innern des Thorax hin, die wir mit den anderen klinischen Untersuchungsmethoden wegen der dicken Pleuraschwarten nicht auffinden konnten. Leider sind wir nicht imstande, für die diesen Schattenbildungen zugrunde liegenden pathologischen Veränderungen eine genügende Erklärung zu geben.

Nach unseren im Vorhergehenden geschilderten Erfahrungen kommen wir zum Schluss über die Röntgenstrahlen als Diagnostikum bei tuberkulösen Lungenerkrankungen zu folgendem Urteil:

Die Röntgenoskopie steht ebenbürtig neben der Auskultation und Perkussion, bald sie übertreffend, bald hinter ihr zurückbleibend (Holzknecht), weil "sie ohne Störung Einblicke in den lebendigen tätigen Organismus gestattet und somit durch die Beteiligung des Auges als Kritiker des Ohrs ein treffliches Mittel objektiver Selbstkritik schafft" (K raft). Wir müssen konstatieren, dass die Röntgendurchleuchtung stets ein positives Resultat ergeben hat, wo wir nach unseren sonstigen Untersuchungsmethoden Tuberkulose als sicher vorliegend annehmen mussten. Hinsichtlich der Frühdiagnose der Lungentuberkulose (sog. "Spitzenkatarrhe") sehen wir uns veranlasst, eine reserviertere Stellung einzunehmen. Nicht in jedem zweifelhaften Falle, wo die anderen physikalischen Methoden versagten, haben wir 
die Röntgenmethode als zuverlässig befunden; sie versagte mitunter ebenso, wie die Auskultation und Perkussion.

Sie bleibt deshalb - vorläufig auch für den Geübteren - in vielen fraglichen Füllen nur eine wertrolle Ergänzung der physikalischen klinischen Untersuchungsmethode. Sie hat sich entschieden bis heute noch nicht zu einer exakten diagnostischen Methode herausgebildet; das Spezifische des Prozesses ist ihr auf jeden Fall unzugänglich. Auch wir sind der Meinung, dass eine nur auf den röntgenologischen Befund aufgebaute Diagnose der Spitzenaffektionen bei fehlendem klinischen Befunde nie mit Sicherheit gestellt werden kann; dagegen sind wir der festen Überzeugung, dass die röntgenologischen Bilder den anatomischen Befunden im allgemeinen näher stehen als die physikalischen Aufschlüsse. Sie gaben entschieden die beste Übersicht über die Ausbreitung des Krankheitsprozesses, gestatteten eine schärfere Beobachtung des Verlaufes und konnten zu einer rechtzeitigen Diagnose bei Beurteilung zweifelhafter Fälle hinsichtlich des Sitzes und der Ausdehnung des erkrankten Bezirkes führen. Wir haben wiederholt feststellen können, dass in Fällen, wo die Untersuchung nur geringe Veränderungen erkennen liess, trotzdem die sonstigen Krankheitssymptome entschieden für ein ausgedehnteres Leiden sprachen, die Röntgenuntersuchung ein wesentlich ungünstigeres, aber zweifellos richtigeres Bild gab, als wir nach der sonstigen Untersuchung erwarten konnten. Sie ist dadurch entschieden ein bedeutungsvolles diagnostisches Hilfsmittel geworden, das für eine grosse Zahl von sonst zweifelhaften Tuberkulosefällen die probatorische Tuberkulininjektion entbehrlich machen kann.

Und wir können unsere Abhandlung in der Hoffnung schliessen, dass gerade für die Frühdiagnose der Lungentuberkulose bei noch mehr verbesserter Technik und vermehrter Übung viel zu erreichen sein wird, so dass die Röntgenuntersuchung in die erste Reihe der Untersuchungsmittel treten wird.

Zum Schlusse gestatten wir uns, dem Chefarzt der Lungenbeilstätten Beelitz, Herrn Dr. Pickert, für die Anregung zu dieser Arbeit, die Überlassung des Materials und das dieser Abhandlung jederzeit entgegengebrachte rege Interesse unseren besten Dank zum Ausdruck zu bringen.

\section{Literaturverzeichnis.}

1. Albers-Schönberg, Der diagnostische Wert der Röntgenuntersuchung en für die innere Medizin. Berlin 1904. 
2. Albers-Schöuberg, Entwickelung des Röntgenverfahrens im Jahre 1901 .

3. Bade, Über den Wert der Röntgenuntersuchung bei der Lungentuberkulose. Fortschr. a. d. G. der Röntgenstr. Bd. V. Heft 3. S. 193.

4. Basch, Der Nachweis der Lungenschwellung und Lungenstarrheit durch Röntgenstrahlen. Wiener med. Wochenschr. 1897. Nr. 5. S. 165.

5. Béclère, Jues rayons de Roentgen et le diagnostic des affections thoraciques Paris 1901. Les actualités médicales.

6. B éclère, $\mathrm{O}$ udin et Barthélem y, Application de la méthode de Roentgen en diagnostic des affections thoraciques et en particulier au diagnostic des lésions de l'appareil respiratoire. Société des Hôp. 25. VI. 1897.

7. Benedikt, M., Das Röntgenlicht im Dienste der inneren Medizin. Kongress für innere Med. Berlin 1897. S. 307.

8. Benedikt, H., Diagnostik innerer Krankheiten durch die Röntgenschen Strahlen. Wiener med. Presse 1898. Nr. 24. S. 961.

9. Bouchard, Les rayons de Roentgen appliqués au diagnostic de la tuberculose pulmonaire. Comptes-rendus. 14. VII. 1896 und Gaz. des Hôp. L. XIX. p. 144 a. 147.1896.

10. Derselbe, Sur l'application de la radioscopie au diagnostic des maladies du thorax. Comptes-rendus. 21. XII. 1896 u. 17. V. 1897.

11. Bouchard et Claude, Les rayons de Roentgen et le diagnostic de la tuberculose. (Congrès pour l'étude de la tuherculose chez l'homme et chez les animaux, 27. Juli bis 2. August 1898 zu Paris.) La semaine médicale 1898. Nr. 42. p. 342 et Gaz. Hôp. 13. VIII. 1898.

12. Brook und Stanley Green, Röntgenstrahlen in der Diagnose der Phthise. Quarterlez Med. Journal, August 1903.

13. Claude et Béclère, Verwendung der Röntgenstrahlen zur Diagnose der Lungentuberkulose. (IV. franz. Kongress zum Studium der Tuberkulose, 1898.) Münch. med. Wochenschr. 1898. Nr. 36. S. 1160.

14. Claude, Application des rayons ${ }_{n} X^{4}$ au diagnostic de la tuberculose pulmonaire. Les rayons $X .1898 . \mathrm{Nr} .26$. p. 772.

15. Coiffier, Rayons $X$ et tuberculose. Les rayons $X .1898$. Nr. 23. p. 6.

16. Colin, Léon, Acad. de méd. 24. X. 1897.

17. Cowl, Thoraxaufnahmen. Zeitschr. f. Diätet. u. phys. Therapie. 1898. Heft 1.

18. Dumstrey und Metzner, Die Untersuchung mit Röntgenstrahlen; eine krit. Studie. Fortschr. a. d. G. d. Röntgenstrahlen. Bd. I. Heft 4. S. 115. 1898.

19. Durr, Spitzeninduration. Comptes-rendus. 124. Nr. 13. 1897.

20. Erpin a $\mathrm{Capo}$, The $\mathrm{X}$ rays in the diagnosis of Tuberculosis. Resista de Medjcina y chirurgia Praticas. 25. Nov. 1898.

21. Gardiner, F., X-Rays as in Diagnostic Agent in Phthisis pulmonalis. The Scottish Medical and Surgical Journal. Nov. 1902.

22. Garrigo u, Radiographies du thorax. Comptes-rendus. 29. XI. 1897.

23. Derselbe, Radiographies rélatives à la taberculose pulmonaire. Académie de Médicine. 3. V. 1898.

24. Gocht, Hanảbuch der Röntgenlehre.

25. G $\mathrm{l} \mathbf{n} \mathrm{mach}$, Über die Diagnoetik innerer Erkrankungen mit Hilfe der Röntgenstrahlen. Wiener med. Wochenschr. 1897. Nr. 36. S. 1650.

26. Derselbe, Über Fortschritte in der Aktinographie. Verh. d. Ges. deutsch. Naturf. u. Ärzte. Düsseldorf 1898. S. 168.

27. Derselbe, Die Radiographie und Radioskopie der inneren Organe. II. internationaler Kongress für med. Elektrologie und Radiologie. Bern 1902. 
28. Grunmach und $W$ ied emann, La radiographie et la radioscopie des organes internes. Arch. d'électricité méd. 1902. p. 717 u. ff.

29. Hildebrand, Über den diagnostischen Wert der Röntgenstrahlen in der inneren Medizin. Münch. med. Wochenschr. 1901. Nr. 49. S. 1957.

30. HoIland, Radiographis of the chest in healty and in phthisis. Lancet 1899. Vol. I. p. 304.

31. Holzknecht, Die Röntgenstrahlen in der inneren Medizin. Fortschr. a.d. G. d. Röntgenstrahlen. Bd. V. Heft 1. S. 67.

32. Derselbe, Ein neves radioskopisches Symptom bei Bronchialstenose und Methodisches. Wiener klin. Rundschan. 1899. Nr. 45.

33. Derselbe, Die Röntgenologische Diagnostik der Erkrankungen der Brusteingeweide. Hamburg 1901.

34. I m m el man n, Kann man mittelst Röntgenstrahlen Lungenschwindsucht schon zu einer Zeit erkennen, in der es durch die bisherigen UntersuchungsMethoden noch nicht möglich ist? Berliner klin. Wochenschr. 1899. Nr. 13. S. 291.

35. Jacobsen, Respiratorische Verschiebung des Mediastinums, ein Symptom einseitiger Bronchostenosen. Berl. med. Ges. 25. III. 1903.

36. Jaworski, Beitrag zur diagnostischen X-Durchstrablung der RespirationsOrgane. Wiener klin. Wochenschr. 1897. Nr. 30.

37. Jicinsky, Diagnose der Tuberkulose mittelst der Röntgenstrahlen. New York. med. Journ. 18. II. 1899. S. 217. (Müach. med. Wochenschr. 1899. Nr. 17. S. 568.)

38. Kelsch und Boinon, Zur frühzeitigen Diagnose der tuberkulösen Brustaffektionen mittelst Radiographie. Académie de Médicine. 21. Dez. 1897.

39: Zraft, Die Röntgenuntersuchung der Brustorgane. Strassburg 1901.

40. Leroux, Charles, Auf welcher Spitze beginnt die Lungentuberkulose?

41. Leo, Über die voraussichtliche Bedeutung der Katbodenstrahlen für die innere Medizin. Berl. klin. Wochenschr. 1896. Nr. 8.

42. Levy-Dorn, Zur Kritik und Ausgestaltung des Röntgenverfahrens. Deutsche med. Wochenschr. 1899.

43. Marco, Die Röntgenstrahlen für die Diagnose der Krankheiten des Respirations- und Zirkulations-Apparates. La clinica Medica italiana. 1897-1898. Nr. 9. Ref. im Zentralbl. f. innere Med. 1899. Nr. 32. S. 839.

44. Marragliano, Application de la radioscopie à l'examen des organes intrathoraciques à l état normal et pathologique. Semaine méd. X. L. II. 50. 1897.

45. Marragliano et C. Caf farena, La radioscopie des pneumoniques. Gazetta degli ospedali e delle clinic 1901. 6. Januar.

46. Martin-Durr, Premières radiographies du poumon malade. Gaz. des Hôp. L. XX. 63. 1897.

47. Mignon, Étude anatomo-clinique de l'appareil respiratoire et de ses annexes par les rayons Roentgen. Gaz. des Hôp. 8. IX. 1898.

48. Derselbe, Lex rayons $X$ dans la tuberculose. IV. Congr. pour l'étude de la tuberculose. Paris 1898.

49. Derselbe, Étude de l'appareil respiratoire par les rayons de Roentgen. Paris 1898.

50. Derselbe, Fitude de la tuberculose poulmonaire par les rayons de Roentgen. Arch. internationales de laryngologie. Paris 1901.

51. Neusser, Die Röntgenstrahlen im Dienste der inneren Medizin. Wiener Briefe, 24. Oktober 1896.

52. Oddo, Anthracose pulmonaire et radiographie. Marseille med. 15, X, 1899. 
150 G. Schellenberg u. A. Scherer: Was leistet die Röntgen-Durchlenchtung etc. [28

53. Rieder, Die Untersuchung der Brustorgane mit Röntgenstrahlen in verschiedenen Durchleuchtungsrichtungen. Fortschr. a. d. G. d. Röntgenstrahlen. Bd. VI. Heft 3. S. 115.

54. Rieder und Rosenthal, Momentaufnahmen des Thorax mit Röntgenstrahlen. Münch. med. Wochenschr. 1899. Nr. 32. S. 1048 und Fortschr. a. d. G. d. Röntgenstrahlen. Bd. III. Heft 3. S. 100.

55. Rosenfeld, Die Diagnostik innerer Krankheiten mittelst Röntgenstrablen. Wiesbaden 1897.

56. Salomonson und Wertheim, Zur Radiograpbie der Lungenkrankheiten. Fortscbr. a. d. G. d. Röntgenstrahlen. Bd. IV. Heft 1. S. 41.

57. Sciallero, Mediastinale und tracheobronchiale Lymphdrïsen. XI. Kongress der ital. Ges. f. innere Med. Pisa, 31. X. 1901.

58. Stubbert, Comparative Diagnosis in pulmonary tuberculosis by the RoentgenRays. New York med. Record. 22. V. 1897.

59. Derselbe, Radiographie bei Untersuchung Brustkranker. Philadelphia med. Journ. 6. I. 1900.

60. Taffier, De la difficulté de localiser les lésions pulmonaires par les signes stéthoseopiques. Société méd. des Hôp. 27. I. 1899.

61. Wals ham, The Rays in prognosis of pulmonary tuberculosis. Lancet 1899 . II. p. 152.

62. Walsham und C. Beale, Die Verwendung der Röntgenographie zur Diagnose von Krankheiten des Thorax. Münch. med. Wochenscbr. 1901. Nr. 8.

63. W as sermann, Zur Diagnose innerer Krankbeiten mittelst Röntgenstrahlen. Wiener klin. Wochenschr. 1899. Nr. 27. S. 439.

64. Weinberger, Atlas der Radiographie der Brustorgane. Wien, E. M. Engel.

65. Derselbe, Über die Untersuchung der Brustkrankheiten mit Röntgenstrahlen. II. internat. Kongr. für med. L'lektrologie u. Radiologie. Bern 1902.

66. W illi a m s, Brusterkrankungen in Röntgenscher Durchleuchtong. 65. Jahresvers. der Brit. Med. Assoziation. Montreal, 31. VIII. bis 4. IX. 1897.

67. Derselbe, The Roentgen-Rays in thoracic diseases. Amer. Jouru. of the med. sciences. Dez. 1897. (Zentralbl. f. Chir. 1898. S. 434.)

68. Derselbe, The X-Rays in thoracic Diagnosis. Amer. Journ, of the med. sciences. Juni 1899.

69. Derselbe, Über den Wert der Untersuchung mit Röntgenstrablen in den Anfangsstadien der Lungentubèrkulose und Röntgenbilder bei Pneumothorax und Pneumohydrothorax. Münch. med. Wochensehr. 9. I. 1900. Nr. 2. S. 59. (Medical News 16. IX. 1899; Philadelphia medical Journal 23. IX. 1899.)

70. Derselbe, Wert der Röntgenstrahlen in der inneren Medizin. Akad. der Med. in New York, 17. I. 1901. (Ref. Münch. med. Wochenschr. 1901. Nr. 9.) 